Ann. Biol. anim. Bioch. Biophys., 1977, 17 (6), 1077-1086.

\title{
Systèmes aminergiques des Gastéropodes pulmonés. II. Mise en évidence au carrefour des voies génitales par la méthode FIF.
}

\author{
par P. BRISSON, J. RÉGONDAUD, J. P. COLLIN \\ avec la collaboration technique de Françoise CHEVALIER
}

Laboratoire de Zoologie et Biologie cellulaire

U. E. R. Sciences fondamentales ef appliquées

40, Avenue du Recfeur Pineau 86022 Poifiers.

Summary. Aminergic systems in pulmonote Gastropoda. II. Fluorescence microscope study in the genital tract carrefour.

Using freeze-drying and formaldehyde treatment (method of Falck et al.), new, welldeveloped catecholaminergic systems have been found in the genital tract of Basommatophora (Planorbarius corneus and Lymnaea stagnalis) and of Stylommatophora (Archachatina marginata and Helix aspersa). The present results deal with the hermaphrodite and female regions of the genital tract.

Basommatophora : green fluorophores were observed in intraepithelial cellular bodies and their corresponding processes and in a more or less large border located at the base of the epithelium of the following parts :

- the wall of the albumen gland duct

- the wall of the fertilization pocket

- the wall of the proximal part of the oviduct

- the vaginal region.

Varicose fibres formed a network around the female genital aperture.

Stylommatophora: green fluorophores were observed at the base of the epithelium of the following parts :

- the fertilization pocket and its duct

- the albumen gland duct

- the oviductary groove.

Its is concluded that two systems may be involved in the production of catecholamines : a) an intrinsic system composed of intraepithelial cells and their processes which might partly or completely constitute the fluorescent border ; b) an extrinsic system, in some regions of the genital tract, composed of the fibres of neurons located in extrinsic ganglions; these fibres might end in the sub-epithelial connective layer.

\section{Introduction.}

Chez les Mollusques, les recherches sur les amines biogènes ont fait l'objet de nombreux travaux (chez les Gastéropodes : voir références in Brisson et Collin, 1977). En nous adressant récemment à un Basommatophore Bulinus truncatus (Audouin), 
nous avons néanmoins mis en évidence trois nouveaux systèmes aminergiques localisés dans : $a$ ) la sole pédieuse, $b$ ) les glandes salivaires et c) l'appareil reproducteur.

Dans ce dernier - que nous avions plus particulièrement étudié - un réseau aminergique apparaît dans la région du vagin et de l'utérus, alors qu'une importante population de cellules aminergiques a pu, en outre, être localisée dans la paroi du canal de la glande à albumine et de son évagination ou poche de fécondation. L'étude, en microscopie électronique, a permis d'identifier dans cette paroi, entre les cellules ciliées banales, des cellules piriformes d'un type particulier - à prolongement basal et contenant de nombreuses vésicules soit claires, soit à cœur dense, caractéristiques des cellules aminergiques. Ce type cellulaire, nouvellement reconnu a été nommé $\mathrm{CA}$ - FIF (le fluorophore - F - vert induit - I - par le_ormaldéhyde - F est significatif de la présence de catécholamine(s) - $\underline{C A}-$ ). Cette terminologie est provisoire et évite de préjuger de la nature neuronale ou non et de la fonction de ce type cellulaire.

Avant d'entreprendre les recherches pharmacologiques visant à établir les éventuelles corrélations fonctionnelles entre les systèmes aminergiques, nouvellement décrits, et la sphère génitale (et/ou tout autre effecteur), il nous a paru important de savoir si l'existence de tels systèmes pouvait être généralisée. Pour cela, nous avons fait appel à quatre représentants de Gastéropodes Pulmonés dont deux Basommatophores et deux Stylommatophores. Cette étude permet, en outre, de dégager le meilleur « modèle » expérimental.

\section{Matériel et méthodes.}

1) Animaux. Nos observations ont porté sur trois espèces de la faune locale récoltées dans la nature ou élevées au laboratoire : deux Basommatophores: la Limnée (Lymnaea stagnalis L.) et la Planorbe (Planorbarius corneus L.), un Stylommatophore autochtone : l'Escargot « petit gris » (Helix aspersa Müller) et un autre, exotique : l'Achatine (Archachatina marginata (Swainson)). Tous les représentants des espèces étudiées étaient matures.

2) Méthode de Falck ef al. (1962), Falck et Owman (1965).

Les animaux débarrassés de leur coquille sont traités, soit « in toto » pour quelques petits spécimens de Planorbes et Limnées, soit par organes pour les autres individus. La méthode de Falck, déjà appliquée à Bulinus, a été exposée antérieurement (Brisson ef Collin, 1977).

Les coupes sériées, de 8 à $12 \mu \mathrm{m}$, ont été observées au microscope Leitz (Orthoplan, orthomat, ploemopak), équipé de filtres BG 12 et $\mathrm{H} 530$ et photographiées sur film Rayoscope Kodak.

Des tesłs de spécificité des monoamines ont été effectués sur Lymnaea, Planorbarius et Archachatina ; les drogues suivantes ont été utilisées :

- Réserpine (Sersapil Ciba) :

- pour les dulçaquicoles, solution à $0.1 \mathrm{mg} / \mathrm{ml}$ dans l'eau d'élevage ; temps d'action $36 \mathrm{~h}$; 
- pour les terrestres, injection de $10 \mathrm{mg}$ de substance active pour $150 \mathrm{~g}$ de poids, répartis en 3 doses sur $48 \mathrm{~h}$, la dernière administrée $24 \mathrm{~h}$ avant le sacrifice.

- Nialamide (Nialamide Pfizer-Clin) utilisé seulement chez les deux espèces limniques à la concentration de $1 \mathrm{mg} / \mathrm{ml}$ du milieu d'élevage; temps d'action $36 \mathrm{~h}$.

Plusieurs individus n'ont pas subi, après lyophilisation, la phase de traitement par le paraformaldéhyde.

Pour faciliter la localisation des cellules aminergiques, les préparations, après observations et photographies en fluorescence, ont été colorées à la trioxyhématéine ferrique.

\section{Résultats.}

Les observations ont porté chez Lymnaea et Planorbarius, sur les parties hermaphrodite et femelle du tractus génital, chez Archachatina et Helix, sur la région du carrefour.

Dans les régions ci-dessous considérées les fluorophores verts (dans les conditions normales : sans traitement pharmacologique préalable des Mollusques), signalant la présence d'amines, apparaissent dans les organes ou individus lyophylisés, exposés aux vapeurs de paraformaldéhyde. De plus, chez les animaux, préalablement traités au nialamide (inhibiteur de la désaminațion oxydative), la fluorescence verte augmente dans les mêmes structures, ce qui indique une rétention accrue des amines dans leurs sites de stockage. A l'opposé, après action de la réserpine ou bien en l'absence de condensation par le paraformaldéhyde, ces mêmes structures aminergiques deviennent imperceptibles.

Ainsi, pour la mise en évidence des systèmes aminergiques par la méthode de Falck ef al:, chez les différentes espèces, nos observations se rapportent donc à des animaux soił étudiés dans les conditions normales, soił prétraités au nialamide.

\section{Chez les Basommatophores}

Les voies génitales ef la région du carrefour de la Planorbe (fig. 1) et de la Limnée (fig. 2) ont été décriłes antérieurement (Holm, 1946 ; Alaphilippe, 1959 ; Alaphilippe, et Régondaud, 1959 ; Duncan, 1960 ; Plesch et al., 1971). Ces auteurs s'accordent pour constater que la partie distale du canal de la glande à albumine présente une ampoule ou chambre de fécondation, disposition retrouvée chez Bulinus (Larambergue, 1939 ; Walter, 1968 ; Wu, 1972 ; Brisson ef Collin, 1977), avant de s'ouvrir dans le cul-de-sac oviductaire (fig. 1).

Dans l'appareil reproducteur, les fluorophores franchement verts apparaissent : a) au voisinage de l'orifice génital femelle et dans la région vaginale sous forme d'un réseau tenu de fibres à varicosités. On peut rappeler que le nerf palléal externe (droił pour Lymnaea forme dextre et gauche pour Planorbarius forme senestre) issu du ganglion pariétal (droit ou gauche) s'anastomose avec le nerf anal provenant du ganglion abdominal ; ces nerfs innervent richement cefte région (Brisson, 1963). Des corps cellulaires, à fluorescence verte, sont disséminés dans la paroi même du vagin, paroi soulignée également par un liséré fluorescent. 


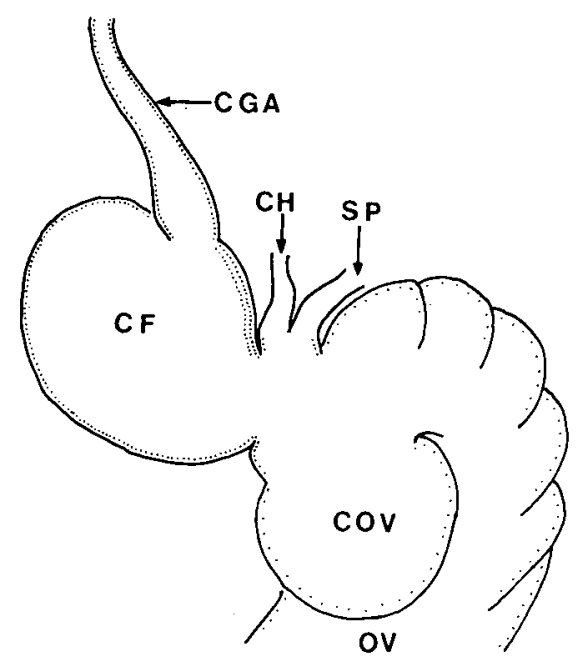

FIG. 1.

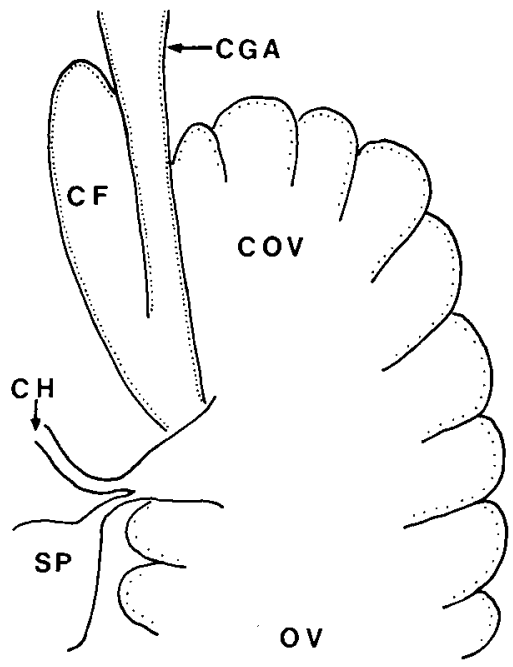

FIG. 2 .

FIG. 1, 2. - Schémas du carrefour des voies génitales de Bulinus truncatus ef Planorbarius corneus (fig. 1) et de Lymnaea stagnalis (fig. 2). (La localisation des fluorophores est indiquée par un pointillé.) Chambre de fécondation, $C F$; canal de la glande à albumine, CGA ; canal hermaphrodite, $\mathrm{CH}$; cul-de-sac oviductaire, COV ; oviducte, OV ; spermiducte, SP.

b) dans l'épithélium des parois du canal de la glande à albumine et de la chambre de fécondation (fig. 3,4 ), jusqu'à son abouchement avec le cul de sac oviductaire. Chez Planorbarius, la fluorescence se manifeste au niveau de cellules piriformes (à pédicule dirigé vers la partie basale de la paroi) nombreuses surtout dans la zone épaissie de la chambre de fécondation, accolée à l'oviducte (fig. 3, 4). La partie la plus externe de ces organes émet une fluorescence trop intense pour permettre, avec notre actuel système optique, l'identification des structures que signalent les fluorophores (fig. 3) (voir discussion).

\section{PLANCHE 1}

FIG. 3, 4. - Coupes passant par la chambre de fécondation (CF) ef le canal de la glande à albumine (CGA) de Planorbarius corneus. $X 120$.

3. - Structures fluorescentes aminergiques ; cs, corps cellulaire.

4. - Coupe au même niveau colorée à la trioxyhématéine.

FIG. 5, 6. - Structures fluorescentes aminergiques dans la paroi oviductaire $(X 120)$ de :

5. - Planorborius corneus.

6. - Lymnaea stagnalis ; cs, corps cellulaire ; ep, épithélium ; I, liséré.

FIG. 7, 8. - Coupes passant par la chambre de fécondation d'Archachatina marginata. $X 80$.

7. - Liséré fluorescent à la base de l'épithélium.

8. - Coupe au même niveau colorée à la trioxyhématéine ; ac, assise conjonctive ; ep, épithélium : I, liséré. 

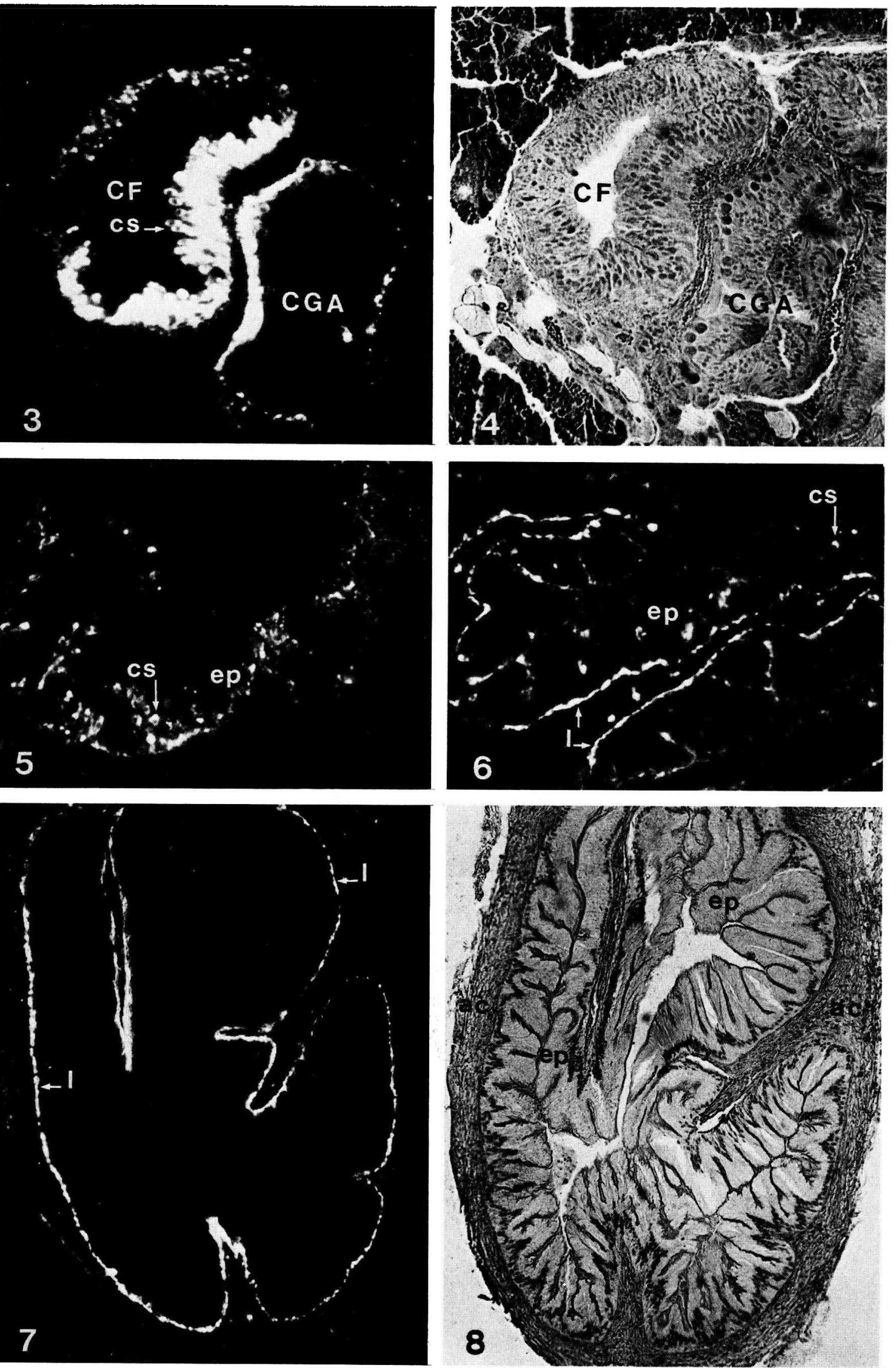
c) dans la paroi épithéliale de la région proximale de l'oviducte chez Planorbarius (fig. 5) et la partie circonvoluée (pars concorta) de Lymnaea (fig. 6), on peut identifier quelques corps cellulaires disséminés et un liséré verts. Ce liséré est plus ou moins développé à la base de l'épithélium.

\section{Chez les Stylommatophores}

Chez les espèces éłudiées, le canal hermaphrodite débouche (fig. 9, 10) dans un diverticule décrit par plusieurs auteurs (Meisenheimer, 1907 ; Perrot, 1937 ; Mead, Mead, 1950 ; Lind, 1973) et qualifié de talon ou de chambre de fécondation, terme que nous adopterons ; cette disposition a été signalée également chez les Arionidae (Perrot, 1939) et les Limacidae (Els, 1974). La chambre de fécondation (fig. 9, 10) est

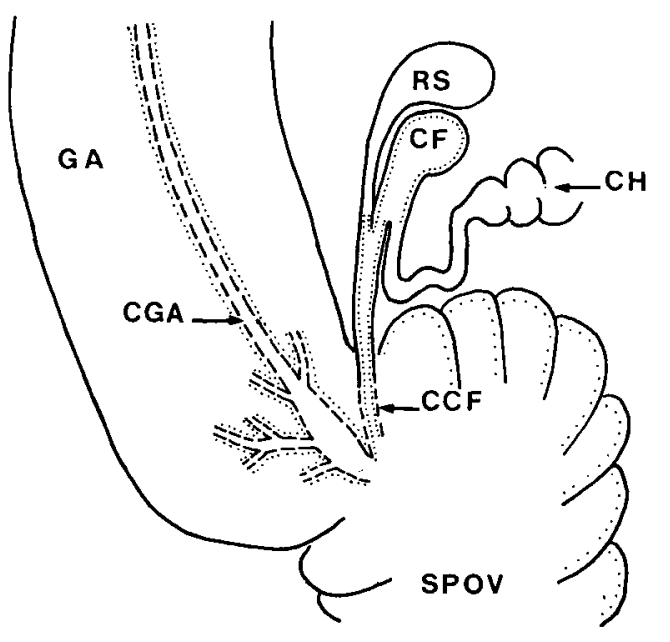

FIG. 9.

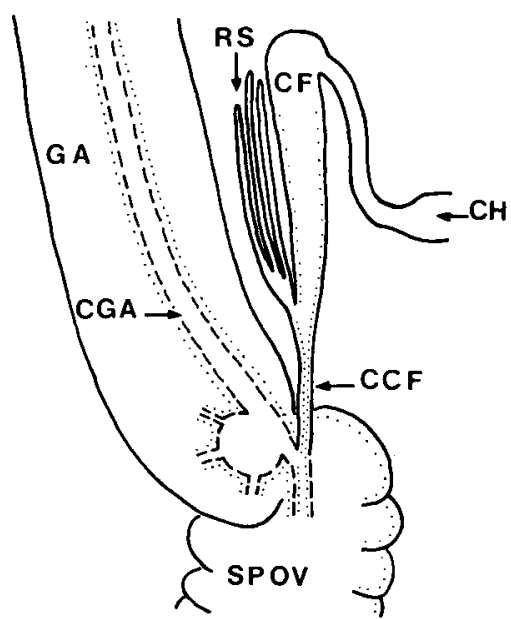

FIG. 10.

FIG. 9, 10. - Schémas du carrefour des voies génitales de Archachatina marginata (fig. 9) et de Helix aspersa (fig. 10). (La localisation des fluorophores est indiquée par un pointillé.)

Canal de la chambre de fécondation, CCF ; glande à albumine, GA ; réceptacle séminal, RS ; spermoviducte, SPOV (Autres légendes comme pour fig. 1, 2).

\section{PLANCHE ||}

FIG. 11, 12. - Coupes passant par le carrefour : canal de la chambre de fécondation (CCF), canal de la glande do albumine (CGA) ef spermoviducte (SPOV) chez Archachatina marginata. X 70 .

11. - Coupe colorée à la trioxyhématéine.

12. - Coupe au même niveau montrant les structures fluorescentes aminergiques.

FIG.13,14. - Structures fluorescenies aminergiques chez Helix aspersa. $X 100$.

13. - Au niveau du canal de la chambre de fécondation (CCF) et du canal de la glande à albumine (CGA).

14. - Au niveau de la gouttière oviductaire ; ac, assise conjonctive ; ep, épithélium. 
prolongée par un canal plus étroit qui, avec le canal de la glande à albumine, se déverse dans le spermoviducte (Duncan, 1975).

Chez Archachatina, à cette chambre de fécondation et la coiffant, aboutit (fig. 9) latéralement une formation recourbée en crosse et qualifiée de réceptacle séminal qui serait le lieu de stockage des spermatozoïdes allochtones.

Les fluorophores verts constituent un liséré continu à la base de l'épithélium des parois de la chambre de fécondation (fig. 7,8 ) ef de son canal, ainsi qu'au départ du réceptacle séminal. Quelques fibres vertes sont disposées concentriquement dans la gaine enveloppant ces organes. Aucune fluorescence n'est décelée dans la paroi du réceptacle, la vésicule séminale ef la partie du canal hermaphrodite aboutissant à la chambre de fécondation. La paroi du canal de la glande à albumine qui reste intraglandulaire (fig. 11) sur toute sa longueur présente une fluorescence verte intense sous-épithéliale (fig. 12). Un réseau, très épais, dans lequel on peut reconnaître quelques corps cellulaires, se trouve à la zone de jonction du canal avec le spermoviducte. Dans le cul-de-sac spermoviductaire, un liséré fluorescent vert souligne la paroi de la gouttière femelle (fig. 12).

Chez Helix aspersa (fig. 10), il y a plusieurs réceptacles séminaux tubulaires (4 à 6) débouchant par un conduit commun dans le canal de la chambre de fécondation. Ils sont emballés dans une gaine conjonctive, commune à celle de la chambre ; cette disposition est comparable à celle décrite chez Helix pomatia (Lind, 1973).

Nous retrouvons un aspect tout à fait comparable à celui observé chez Archachatina : fluorescence verte au niveau des parois de la chambre de fécondation et de son canal (fig. 13), dans la paroi du canal de la glande à albumine jusqu'à son débouché, et dans la paroi de la gouttière oviductaire (fig. 14). Aucune fluorescence n'est décelée dans les réceptacles séminaux et le canal hermaphrodite.

\section{Discussion.}

['étude de certaines régions de l'appareil génital de plusieurs Gastéropodes Pulmonés nous permet d'identifier, par la méthode de Falkc ef al., des systèmes monoaminergiques bien développés demeurés jusqu'ici inconnus. La comparaison d'organes lyophilisés puis soumis aux vapeurs de formaldéhyde (animaux témoins) avec ceux, traités au préalable par des drogues, ou encore avec ceux non condensés par l'aldéhyde, permet de garantir, comme chez Bulinus (voir discussion Brisson et Collin, 1977), que la fluorescence signale l'existence de moncamines. La présence de ces fluorophores verts correspond à un stockage probable de catécholamines (Brisson et Collin, 1977). La durée de la condensation $(2 \mathrm{~h})$ nous avait conduit à suggérer l'existence de dopamine et/ou de noradrénaline ; la dopamine est une amine très fréquente chez les Mollusques (Welsh, 1972 ; Pentreath ef Cottrell, 1974). De plus, l'histamine, la tyrosine, la phénylalanine ef des amines (4-hydroxylées) rencontrées chez les Mollusques comme la tyramine ef l'octopamine (ref. in Brisson et Collin, 1977) ne donnent pas de produits de condensation (Björklund ef al., 1975) dans les conditions où nous avons appliqué la technique de Falck et al. L'étude microspectrofluorimétrique comblera très prochainement cette lacune, relative à l'identification précise des amines à différents niveaux du tractus génital (Coll. H. G. Hartwig, Giessen). 
Trois des espèces étudiées sont autochtones et soumises dans la nature à des rythmes saisonniers très marqués. Les animaux observés étaient tous en phase d'activité reproductrice ; des recherches ultérieures, par des techniques appropriées, pourraient permettre de déceler les variations saisonnières éventuelles du taux des monoamines.

Chez les Stylommatophores, les éléments CA-FIF (voir introduction) ont été mis en évidence au niveau de la glande à albumine, de la chambre de fécondation ef d'une partie du spermoviducte. En comparant ces résultats à ceux, également originaux, obtenus chez Bulinus (Brisson et Collin, 1977), on constate que les fluorophores sont localisés dans des organes ayant reçu des dénominations semblables. Compte tenu de la terminologie utilisée par les différents auteurs eł de l'existence d'amines, l'idée d'organes à fonction analogue ou voisine est à prendre en considération. Faute de recherches embryologiques, l'homologie ne peut être garantie.

Si chez les Basommatophores, les cellules fluorescentes sont en général facilement repérables en microscopie photonique après coloration et présentent pour certaines un prolongement basal, chez Archachatina ef Hélix par contre, nos recherches n'ont pas encore permis d'identifier, avec certitude, les cellules signalées par les fluorophores. La poursuite de ces recherches, à l'aide de la technique radioautographique à haute résolution et de drogues permettra de préciser la structure et la nature neuronale (ou non) de ces types cellulaires, les sites de synthèse, de stockage et de libération aminergiques.

En résumé, il nous semble probable que les catécholamines de l'appareil génital sont élaborées par deux types d'éléments, intrinsèques et extrinsèques :

- Les premiers, correspondent aux corps ef prolongements cellulaires intraépithéliaux, dont la nature neuronale n'a pu, jusqu'ici, être définitivement démontrée tout au moins chez Bulinus (Brisson et Collin, 1977). Ces prolongements dont le trajet précis ne peut être établi par la méthode de Falck, pourraient constituer, en totalité ou en partie, le liséré très fluorescent soulignant la région basale des épithéliums concernés.

- Les seconds (dans certaines régions du tractus génital comme par exemple celles du vagin et du pore génital femelle des Basommatophores) pourraient se rapporter à des fibres issues de neurones localisés dans des ganglions extrinsèques, ces fibres aboutissant dans l'assise conjonctive ou conjonctivo-musculaire sous-épithéliale.

Les prolongements cellulaires intrinsèques et extrinsèques pourraient, éventuellement, s'intriquer au niveau de certaines régions du tractus génital.

Les études ultérieures, visant à la destruction chirurgicale ef chimique de ces systèmes monoaminergiques devaient permettre de dissocier les constituants intrinsèques et extrinsèques. Le rôle de ces systèmes (discuté antérieurement à propos de Bulinus : Brisson et Collin, 1977) est encore hypothétique. Nos observations, chez quatre autres genres de Gastéropodes, nous permettent de généraliser leur existence ef font ressortir que l'organisation du carrefour des voies génitales présente, chez les Pulmonés, une certaine homogénéité. Dans l'état actuel de nos investigations les Basommatophores nous paraissent constituer un matériel plus particulièrement propice à l'étude physiologique que nous entreprenons. 
Remerciements. - Cette étude a été réalisée avec l'appui financier du CNRS. Les Laboratoires Ciba (Suisse) et Pfizer-Clin (France) nous ont gracieusement fourni les drogues.

\section{References}

ALAPHILIPPE F., 1959. Contribution à l'étude anatomique et histologique des voies génitales de Planorbis (Plonorbarius) corneus L. (Gastéropode Pulmoné). Bull. Biol. Fr. Belg., 93, 260-287.

ALAPHILIPPE F., REGONDAUD J., 1959. Contribution à l'étude du carrefour des voies génitales chez Planorbis (Planorbarius) corneus L. et Limnaea stagnalis L. Bull. Soc. Zool. Fr., 84, 485-493.

BJORKLUND A., FALCK B., LINDVALL O., 1975. Microspectrofluorometric analysis of cellular monoamines after formaldehyde or glyoxylic acid condensation, 249-294. In BRADLEY P. B., Methods in brain research, Wiley and sons, London.

BRISSON P., 1963. Asymétrie des muscles rétracteurs du bulbe buccal et des centre nerveux chez les Gastéropodes Pulmonés. Bull. Soc. Zool. vr., 88, 432-448.

BRISSON P., COLLIN J. P., 1977. Systèmes aminergiques des Gastéropodes Pulmonés : I. Ełude en microscopie de fluorescence et électronique des cellules aminergiques du canal de la glande à albumine chez Bulinus truncatus Audouin. Biol. Cell. (sous presse).

DUNCAN C. J., 1960. The genital systems of the freshwater Basommatophora. Proc. zool. Soc. Lond., 135, 339-356.

DUNCAN C. J., 1975. Reproduction, 309-365. In : FRETTER V., PEAKE J., Pulmonates, Acad. Press.

ELS A., 1974. The morphology and histology of the genital system of the pulmonate Milax gagates (Drap.). Ann. Univ. Von Stellenbosch., 49, 1-39.

FALCK B., HILLARP N. A., THIEME G., TORP A., 1962. Fluorescence of catecholamines and related compounds condensed with formaldehyde. J. Histochem. Cytochem., 10, 348-354.

FALCK B., OWMAN Ch., 1965. A detailed methodological description of the fluorescence method for the cellular demonstration of biogenic monoamines. Acia Univ. Lund, 7, 1-23.

HOLM L. W., 1946. Histological and functional studies on the genital tract of Lymnaeo stagnalis appressa Say. Trans. amer. Micros. Soc. Menasha, 65, 45-68.

LARAMBERGUE M. de, 1939. Etude de l'autofécondation chez les Gostéropodes Pulmonés. Recherche sur l'aphallie et la fécondation chez Bulinus (Isidora) contortus Mich. Bull. Biol. Fr. Belg., 73, 19-231.

LIND H., 1973. The functional significance of the spermatophore and the fate of spermatozoa in the genital tract of Helix pomatia (Gastropoda Stylommatophora). J. Zool. Lond., 169, 39-64.

MEAD A. R., 1950. Comparative genital anatomy of some african Achatinidae (Pulmonata). Bull. Mus. comp. Zool. Harv., 105, 219-291.

MEISENHEIMER J., 1907. Biologie, Morphologie und Physiologie des Begatungsvorganges und der Eiablage von Helix pomatio. Zool. Jb., 25, 461-502.

PENTREATH V. W., COTTRELL G. A., 1974. Ultrastructure of a giant dopamine containing neurone in Plonorbis corneus. Experientia, 3, 293-294.

PERROT J. L., 1937. La chambre de fécondation de l'Helix pomatio et la présence de spermatozoïdes à son niveau. C. R. Acad. Sci. Paris, 204, 1216-1218.

PERROT J. L., 1939. Contribution à l'étude de la fécondation chez les Pulmonés Stylommatophores. Le cas de l'Arion empiricorum Fer. (Arion rufus L.). Bull. Biol. Fr. Belg., 73, 408-432.

PLESCH B., JONG-BRINK M. de, BOER H. H., 1971. Histological and histochemical observations on the reproductive tract of the hermaphrodite pond smail Lymnaea stagnalis L. Nether. J. Zool., 21, 180-201.

WALTER H. J., 1968. Morphological features of liberian Bulinus and B. truncalus of Egypt : a pictorial essay on snails of three subgenera (Planorbidae Basommatophora). Malacol. Rev., 1, 35-89.

WELSH J. H., 1972. Mollusca, 91-99. In : BLASCHKO H., MUSCHOLL E., Handbuch der experimentellen Pharmakologie, Springer Verlag, Berlin.

WU S. K., 1972. Comparative studies on a polyploïd series of the African snail genus Bulinus (Basommatophora : Planorbidae). Malacol. Rev., 5, 95-164. 